\title{
La facilitación comercial y sus efectos en el comercio bilateral chileno de 2006 a 2014
}

\author{
Darcy Fuenzalida-O’Shee, Bárbara Valenzuela-Klagges \\ y Alejandro Corvalán-Quiroz
}

\section{Resumen}

En este estudio se analizan los efectos de la facilitación del comercio en las exportaciones chilenas durante el período 2006-2014. Se revisarán las evidencias y los estudios sobre la facilitación del comercio y sus efectos, principalmente en América del Sur, y se analizará el patrón exportador chileno. Posteriormente, se estimará un modelo gravitacional ampliado relativo a flujos del comercio exterior bilateral chileno con 89 países, para medir el efecto de la facilitación del comercio durante el período analizado. Dicha estimación se realizará mediante datos de panel estáticos con efectos fijos, aleatorios y dinámicos. Los principales resultados confirman que la estimación que mejor describe el comportamiento exportador chileno es aquella en la que se emplean datos de panel estático con efectos aleatorios y dinámicos con dos retardos de la variable dependiente exportación, y determinan que los costos de exportación por contenedor tienen un efecto negativo en este flujo comercial.

\section{Palabras clave}

Liberalización del intercambio, facilitación del comercio, importaciones, exportaciones, costos, política comercial, convenios comerciales bilaterales, estadísticas comerciales, Chile

\section{Clasificación JEL}

F140, F150, F170

\section{Autores}

Darcy Fuenzalida-O'Shee es Rector y Académico del Departamento de Ingeniería Comercial de la Universidad Técnica Federico Santa María, Chile. Correo electrónico: darcy.fuenzalida@usm.cl.

Bárbara Valenzuela-Klagges es Investigadora externa en el Instituto de Economía Internacional de la Universidad Jaume I, España. Correo electrónico: barbara. valenzuelak@usm.cl.

Alejandro Corvalán-Quiroz es Académico del Departamento de Industria y Economía de la Facultad de Ingeniería en la Universidad de Playa Ancha de Ciencias de la Educación, Chile. Correo electrónico: alejandro.corvalan@upla.cl. 


\section{Introducción}

Un proceso de liberalización o integración comercial exitosa se inicia con la capacidad de un país de mover mercancías a través de las fronteras de manera confiable, rápida y con costos bajos. Aun cuando el costo del transporte terrestre, aéreo o marítimo, así como los aranceles o las gestiones aduaneras y los servicios logísticos, no cambien los fundamentos teóricos de las ventajas comparativas o los beneficios del comercio internacional, pueden distorsionar el movimiento de los bienes o servicios e influir en la orientación del comercio, los términos de intercambio, los acuerdos entre países y la competitividad global. En este sentido, los costos de transacción pueden diferir según la eficiencia, la integridad del exportador y las entidades públicas y privadas que intervienen en la operación exportadora e importadora (OCDE, 2003).

Jaén (2010) plantea que, en lo que respecta a la facilitación del comercio, el transporte eficiente y los servicios logísticos han emergido como elementos estratégicos para explicar el acceso a los mercados, y señala lo siguiente: "La facilitación del comercio se refiere a aspectos relacionados con un entorno más predictible, seguro y eficiente del comercio internacional, a través de la simplificación, estandarización y armonización de las formalidades administrativas" (Jaén, 2010, pág. 108). Helble, Shepherd y Wilson (2007) añaden que las ganancias de una mayor predictibilidad derivada de la facilitación del comercio pueden percibirse mediante la disminución de los costos comerciales y el aumento de las ganancias internas.

En variados estudios (Batra, Kaufmann y Stone, 2003; Wilson, Man y Otsuki, 2003; Engman, 2005; Nordas y otros, 2006; Wilson, 2007; Jean-François y otros, 2007; Echeverría, 2007; Hummels, 2007; Iwanow y Kirkpatrick, 2007; Martínez-Zarzoso y Márquez-Ramos, 2008; Servín, 2008; Castro, 2010; Djankov, Freund y Pham, 2010; Portugal-Pérez y Wilson, 2012; Moïsé y Sorescu, 2013; Cordero, 2014; Jordaan, 2014; Sant'Anna y De Souza, 2014; Zaki, 2015) se reconoce que las medidas de facilitación del comercio, entre ellas los sistemas logísticos en general, tienen un efecto positivo en los flujos comerciales de los países y, por ende, en su productividad. Al respecto, Djankov, Freund y Pham (2010) estimaron que un día adicional de demora antes de embarcar reduciría el comercio en por lo menos un 1\%. La OCDE (Wilson, 2007) señala que los países con menores costos de transacción han experimentado un mayor aumento de su producto interno bruto a partir de un incremento de las exportaciones de manufacturas. Más aún, las pequeñas reducciones de los costos de transacción incrementan significativamente los flujos del comercio. En concordancia, Jean-François y otros (2007) plantean que los países en desarrollo pueden aprovechar el comercio mundial aplicando medidas de facilitación del comercio y sistemas logísticos, y sugieren avanzar en la infraestructura de transporte y en la aplicación de las tecnologías de la información aduanera. En el estudio de Iwanow y Kirkpatrick (2007) se concluye que un progreso del 10\% en la facilitación del comercio produciría un aumento aproximado del 5\% en las exportaciones. Martínez-Zarzoso y Márquez-Ramos (2008) observan que los flujos comerciales aumentan al reducir los costos del transporte y el número de días necesarios para el comercio. Moïsé y Sorescu (2013) concluyen que ofrecer información relacionada con el comercio, simplificar y armonizar la documentación, y automatizar los procedimientos tiene mayor impacto en los volúmenes y los costos del comercio de un país. Jordaan (2014) se basa en la realidad sudafricana y concluye que mejorar el entorno aduanero del país importador proporciona ganancias mayores en términos del aumento de los flujos de comercio, seguido por la mejora del entorno normativo y la infraestructura nacional. Sant'Anna y De Souza (2014) analizan el comercio exterior brasileño y confirman que, si aumenta la facilitación comercial, también lo hacen las exportaciones brasileñas. Zaki (2015) muestra que una multitud de variables de facilitación del comercio, entre ellas Internet, la burocracia y la corrupción, afectan los tiempos de transacción para importar y exportar, y concluyen que la facilitación y la liberalización del comercio son complementos en lugar de sustitutos. 
Dadas las evidencias, Moreira y otros (2013) consideran preocupante que tanto los responsables de formular las políticas como los investigadores de América Latina no consideren la dimensión distributiva de los costos del comercio derivados de la facilitación comercial. Dado lo expuesto y el interés por analizar el comercio exterior chileno en el contexto global y específico relacionado con los costos del comercio, el objetivo de este estudio es analizar los efectos de la facilitación del comercio en las exportaciones chilenas durante el período que va de 2006 a 2014.

Con ese fin, se revisarán las evidencias y los estudios sobre la facilitación del comercio y sus efectos en América del Sur, y se analizará el patrón exportador chileno, con el propósito de comprender la realidad actual y las oportunidades que se pueden alcanzar al reducir los costos internos derivados del proceso exportador o importador. Posteriormente, se estimará un modelo gravitacional ampliado relativo a los flujos del comercio exterior bilateral chileno con 89 países, para medir el efecto de la facilitación del comercio durante el período en estudio. Dicha estimación se realizará mediante datos de panel estáticos con efectos fijos, aleatorios y dinámicos.

En los últimos tiempos se ha incrementado la cantidad de artículos en los que se aplica el modelo gravitacional para determinar el comportamiento exportador, y en los que se incluye, de forma directa y significativa, a Chile o a países del Cono Sur, el Mercado Común del Sur (MERCOSUR) o la Comunidad Andina (CAN) (Durso y Ochoa, 2003; Giacalone, 2003; Kamil y Ons, 2003; Martínez-Zarzoso y Nowak-Lehmann, 2003; Nicita, Olarreaga y Soloaga, 2003; Vallejo y Aguilar, 2004; Cárdenas y García, 2004; Lewer y Sáenz, 2004; Martínez-Zarzoso y Suárez-Burguet, 2004; Lara y Soloaga, 2005; López y Fernando, 2005; Sandberg, Seale y Taylor, 2006; Sá Porto y Azzoni, 2007; Serrano y Pinilla, 2008; Valenzuela-Klagges, 2011; Ramos-Martínez y otros, 2012; Álvarez, Fischer y Natera, 2013; Florensa y otros, 2013; Bacaria-Colom, Osorio-Caballero y Artal-Tur, 2013; Hernández, 2014; Valenzuela-Klagges y Espinoza-Brito, 2015). En varios de estos estudios se utiliza el corte transversal para aplicar este modelo, pero en la actualidad se observa una tendencia a estimar el modelo gravitacional mediante datos de panel estáticos con efectos fijos o aleatorios (De Souza y Burnquist, 2011, y Sant'Anna y De Souza, 2014), y dinámicos. Sin embargo, siguen siendo escasos los estudios cuyo propósito es determinar los efectos de la facilitación comercial en el comercio de América del Sur, y no se registran publicaciones relativas al comercio exterior chileno.

Este estudio tiene por objeto aportar al debate científico y en él se abarcan problemáticas actuales de América Latina (Banco Mundial, 2013a y 2013b). Los principales resultados confirman que la estimación de datos de panel estático con efectos aleatorios y dinámicos con dos retardos de la variable dependiente exportación es la que mejor describe el comportamiento exportador e importador chileno y permite determinar que los costos de exportación por contenedor tienen un efecto negativo en este flujo comercial.

\section{Facilitación del comercio y realidad de América del Sur}

Para Izam (2001), la facilitación del comercio se relaciona con una gran diversidad de ámbitos referidos a las transacciones internacionales de bienes o servicios, así como al movimiento de capital y de personas de negocios, y abarca temas como los asuntos aduaneros, las normas técnicas y los estándares de calidad, las medidas sanitarias y fitosanitarias, el transporte (terrestre, aéreo, marítimo, lacustre, fluvial y ferroviario), la valoración aduanera, la infraestructura, la propiedad intelectual, los servicios vinculados al comercio, la tributación, las reglas de origen, la libertad de tránsito, la transmisión electrónica de información comercial, la atención que se brinda a los hombres de negocios y a los pasajeros, los procedimientos aduaneros, la transparencia, el control y la simplificación, el comercio electrónico, los servicios asociados a los pagos y a los seguros relacionados con las transacciones internacionales e, 
incluso, las cuestiones arancelarias y no arancelarias. Por otra parte, al asociar la facilitación del comercio con la modernización de las aduanas y las agencias gubernamentales vinculadas a la inspección en frontera, Echeverría (2007) añade a la enumeración anterior las alianzas público-privadas, el acceso rápido a la información, el transporte eficiente e integrado, y los trámites cortos y poco engorrosos.

Sobre la base de estos ámbitos temáticos de la facilitación comercial, se podrían destacar diversos estudios de los últimos años, como el de Wilson, Otsuki y Man (2004), el de Shepherd y Wilson (2006), el de Helble, Shepherd y Wilson (2007), el de Márquez-Ramos y otros (2007), el de Iwanow y Kirkpatrick (2009), el de Hesketh (2010), el de DjanKov, Freund y Pham (2010), el de Márquez-Ramos y otros (2011), el de Zamora y Sierens (2014), el de Ueki (2015) y el de Shepherd (2016). Estos trabajos pueden subdividirse según los siguientes temas de estudio:

- $\quad$ Rutas de acceso por carretera y distancia mínima de conexión. Shepherd y Wilson (2006) presentan una base de datos correspondiente a 138 ciudades de 27 países de Europa y Asia Central. Demuestran que la calidad de las redes de carreteras afecta positivamente los flujos comerciales intrarregionales y que mejorarlas podría aumentar el comercio en un 50\%.

- $\quad$ Costos de transporte marítimo y servicios logísticos. Márquez-Ramos y otros (2007 y 2011) concluyen que el descenso del costo de los fletes y las tasas de carga bajas podrían facilitar las exportaciones. Además, observan que, cuanto mayor es el tamaño de un puerto, menor es el costo del flete, y que, cuanto mayor es el número de líneas de transporte marítimo que compiten en una ruta, menores son las tasas de carga. En el estudio de Zamora y Sierens (2014) se consideran las aduanas, el transporte internacional y los servicios logísticos, y se observa que estas tres variables inciden directamente y de manera significativa en la competitividad logística del comercio internacional.

- $\quad$ Reducción de aranceles. En el estudio de Hesketh (2010) se plantea que una reducción de los aranceles y las barreras no arancelarias al comercio mundial puede fomentar un mayor uso de la tecnología de energía baja en carbono.

- Uso de tecnologías. DjanKov, Freund y Pham (2010), Helble, Shepherd y Wilson (2007), y Wilson, Otsuki y Man (2004) consideran el índice de disposición respecto del gobierno electrónico.

- $\quad$ Obstáculos al comercio en general. Ueki (2015) investiga el impacto de los costos del comercio en la propensión a exportar y en la intensidad de las empresas en el sudeste de Asia y América Latina. Utiliza encuestas dirigidas a empresas del Banco Mundial y concluye que los obstáculos al comercio no afectan significativamente la intensidad exportadora de estas últimas.

A partir del año 2014, se avanza en la facilitación comercial mundial al firmarse el Acuerdo sobre Facilitación del Comercio entre los países miembros de la Organización Mundial del Comercio. Por otra parte, Shepherd (2016) observa que el Foro de Cooperación Económica Asia-Pacífico (APEC) continúa orientándose hacia la facilitación del comercio y sugiere analizar los países que se destacan por una marcada reducción de costos en la transacción internacional, como es el caso de la Provincia China de Taiwán. El autor identifica tres áreas generales para mejorar la facilitación comercial en los países miembros de la APEC: la conectividad física, la conectividad institucional y la conectividad entre los pueblos.

En el cuadro 1 se exponen diez artículos que evidencian la realidad de América Latina y el Caribe en los últimos años. En nueve de ellos se destacan la excesiva documentación y los procedimientos aduaneros ineficientes; en ocho se sugiere la necesidad de renovar y aumentar la infraestructura portuaria, terrestre y vial en general; en cuatro se analiza la gestión pública y la necesidad de hacer reformas modernistas que garanticen la facilitación del comercio sin afectar la seguridad; en tres se plantea la preocupación por los días de diligencias o embarque necesarios para exportar; en dos se expone la necesidad de implementar técnicas de información, y en uno se incorpora la gestión privada. No se registran estudios de facilitación comercial que se centren en Chile. 
Cuadro 1

Revisión de evidencia en países de América Latina y el Caribe

\begin{tabular}{|c|c|c|c|}
\hline Autor & Año & Países en estudio & Conclusiones y sugerencias \\
\hline $\begin{array}{l}\text { Batra, Kaufmann } \\
\text { y Stone }\end{array}$ & 2003 & América Latina & $\begin{array}{l}\text { El tiempo promedio que se necesita para diligenciar un proceso de exportación o } \\
\text { importación en América Latina oscila entre } 2 \text { y } 48 \text { días, mientras que en otros países } \\
\text { oscila entre } 1 \text { y } 24 \text { días. }\end{array}$ \\
\hline $\begin{array}{l}\text { Ueki, Tsuji y } \\
\text { Cárcamo }\end{array}$ & 2005 & América Latina & $\begin{array}{l}\text { Los procedimientos onerosos relacionados con el comercio constituyen barreras } \\
\text { considerables para las pymes que desean exportar sus productos. El manejo ineficiente } \\
\text { de los documentos comerciales supervisados por departamentos gubernamentales } \\
\text { aumenta el costo del comercio internacional. Se hacen las siguientes recomendaciones } \\
\text { a la región: establecer una red de investigadores para observar las pymes; ofrecer } \\
\text { educación a distancia sobre comercio e industria; formar conglomerados virtuales; } \\
\text { construir infraestructura de telecomunicaciones comerciales; unificar los estándares } \\
\text { tecnológicos y la seguridad de las TIC, y facilitar el comercio. }\end{array}$ \\
\hline Nordas y otros & 2006 & República Dominicana & $\begin{array}{l}\text { Se aprecia la relevancia de los sistemas logísticos en general y de las medidas de } \\
\text { facilitación del comercio en particular. }\end{array}$ \\
\hline Servín & 2008 & $\begin{array}{l}\text { Paraguay y Unión } \\
\text { Europea }\end{array}$ & $\begin{array}{l}\text { Se recomienda establecer compromisos para impulsar mecanismos que faciliten el } \\
\text { comercio exterior entre el Paraguay y la Unión Europea, en particular modernizar los } \\
\text { puertos, desarrollar las técnicas de la información y la comunicación, y simplificar y } \\
\text { agilizar los procedimientos aduaneros. }\end{array}$ \\
\hline Castro & 2010 & $\begin{array}{l}\text { Centroamérica } \\
\text { y Panamá }\end{array}$ & $\begin{array}{l}\text { Se destacan los siguientes problemas: tiempos e inspecciones excesivos para obtener } \\
\text { permisos especiales, limitada institucionalidad de apoyo, problemas de capacitación } \\
\text { del personal de aduanas, procedimientos y sistemas excesivos, y deficiencias en } \\
\text { infraestructura. Como parte de las acciones de mejora se hace énfasis en que se } \\
\text { deben implementar sistemas de transmisión electrónica y revisar los procedimientos } \\
\text { para simplificar los trámites. Además, se hacen recomendaciones para fortalecer la } \\
\text { institucionalidad de apoyo a las mipymes. }\end{array}$ \\
\hline Barbero & 2010 & América del Sur & $\begin{array}{l}\text { Se identifican acciones prioritarias para mejorar la facilitación del comercio: aumentar } \\
\text { la provisión de infraestructura básica y servicios de infraestructura dirigidos más } \\
\text { directamente a la logística; mejorar los servicios que brinda el Estado por sí mismo, en } \\
\text { particular la gestión aduanera y paraaduanera; mejorar el desempeño de los privados } \\
\text { con capacitación a las empresas de menores dimensiones, e impulsar políticas de } \\
\text { calidad en el desempeño logístico. }\end{array}$ \\
\hline Stark & 2011 & $\begin{array}{l}\text { El Salvador y } \\
\text { Guatemala }\end{array}$ & $\begin{array}{l}\text { Se destaca la necesidad de formular una estrategia de inserción internacional que } \\
\text { permita dar coherencia a los programas y las acciones en el marco de una visión de } \\
\text { país de largo plazo, compartida por los principales actores nacionales. Dicha estrategia } \\
\text { debe sustentarse en la innovación y la creación permanente de bienes y servicios } \\
\text { nuevos y de calidad, así como en el incremento de la productividad. Los esfuerzos } \\
\text { para facilitar el comercio deben insertarse en estos procesos. }\end{array}$ \\
\hline $\begin{array}{l}\text { Fuentes y Del } \\
\text { Castillo }\end{array}$ & 2012 & México & $\begin{array}{l}\text { Los resultados muestran que la política de reducción arancelaria propuesta no tiene } \\
\text { efecto significativo sobre el crecimiento económico ni sobre la distribución del ingreso } \\
\text { por clase social. La política de reducción arancelaria solo implica un cambio en los } \\
\text { componentes de la balanza comercial y en la composición del empleo. }\end{array}$ \\
\hline Cordero & 2014 & $\begin{array}{l}\text { CARICOM y } \\
\text { Centroamérica }\end{array}$ & $\begin{array}{l}\text { La complementariedad entre Centroamérica y la Comunidad del Caribe (CARICOM) es } \\
\text { factible gracias a la especialización productiva, pero se requiere crear rutas de acceso } \\
\text { para mantener la competitividad. Además, es necesario avanzar en la facilitación } \\
\text { comercial con el propósito de reducir los costos y el tiempo de importación y exportación. }\end{array}$ \\
\hline Cortes & 2014 & Colombia & $\begin{array}{l}\text { Colombia no es un país competitivo en materia comercial debido al rezago de su } \\
\text { organización logística, principalmente en aspectos relacionados con la infraestructura, } \\
\text { la trazabilidad, el régimen aduanero, la corrupción y la seguridad, lo que aumenta } \\
\text { los costos de exportación. }\end{array}$ \\
\hline
\end{tabular}

Fuente: Elaboración propia.

Se seleccionaron 104 países cuyas exportaciones e importaciones con Chile se mantuvieron continuas en el período que va de 2006 a 2014. Treinta y tres de ellos pertenecen a Europa, 18 a África, 15 a Asia, 13 a Oriente Medio, 13 a América del Norte y el Caribe, 9 a América del Sur y 3 a Oceanía. En 2010, en estos 104 países se exigían en promedio siete documentos para importar (véase el gráfico 1, que expone 132 países, incluidos los 104 del estudio). Chile se ubicaba por debajo del promedio, con seis documentos. Entre los países en los que se exigía un menor número de documentos para importar o exportar se destacan Francia, con dos, Suecia y la República de Corea, con tres, y el Canadá, con cuatro. Entre los países en los que se exigía un mayor número de documentos para importar se destacan el Camerún y Kasajstán (12), Rusia (11), y Camboya, Laos, Mozambique, el Paraguay y Puerto Rico (10) (Banco Mundial, s/f). Entre 2012 y 2014, Chile se destaca en América del Sur al disminuir a cinco la cantidad de documentos que se exigen para exportar. En contraste, en el Paraguay y la República Bolivariana de Venezuela se exigen nueve. 


\section{Gráfico 1}

Número de documentos exigidos para importar en 132 paísesª 2010

(En número de documentos y países)

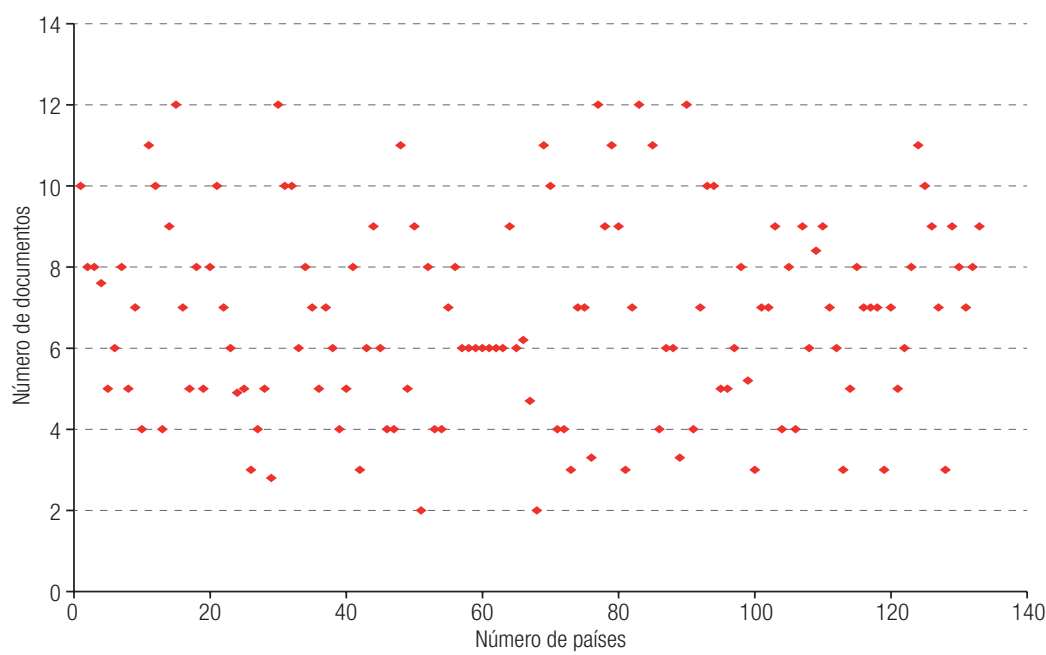

Fuente: Elaboración propia, sobre la base de datos de Banco Mundial, "Datos de libre acceso del Banco Mundial" [en línea] https://datos.bancomundial.org.

a Países cuyas exportaciones e importaciones con Chile se mantuvieron continuas en el período que va de 2006 a 2014.

El promedio de costo de exportación de un contenedor en los 104 países seleccionados para este estudio ascendía a 1.092,00 dólares en 2010 (véase el gráfico 2). Chile se ubicaba por debajo del promedio, con 745 dólares. Entre los países con menor costo de exportación por contenedor se destacan los países asiáticos, sobre todo Singapur (456 dólares), Malasia (450 dólares) y China (500 dólares), mientras que los países con mayor costo de exportación por contenedor son Kasajstán (3.005 dólares), Zambia (2.664 dólares) y la República Bolivariana de Venezuela (2.590 dólares). En 2013 y 2014 se destaca nuevamente Singapur, donde se logra bajar este costo a 440 dólares, mientras que en la República Bolivariana de Venezuela y Zambia se observa un incremento considerable a 3.490 dólares y 6.369 dólares, respectivamente.

Gráfico 2

Costos de exportación en 132 países $^{\mathrm{a}}$, 2010

(En dólares y países)

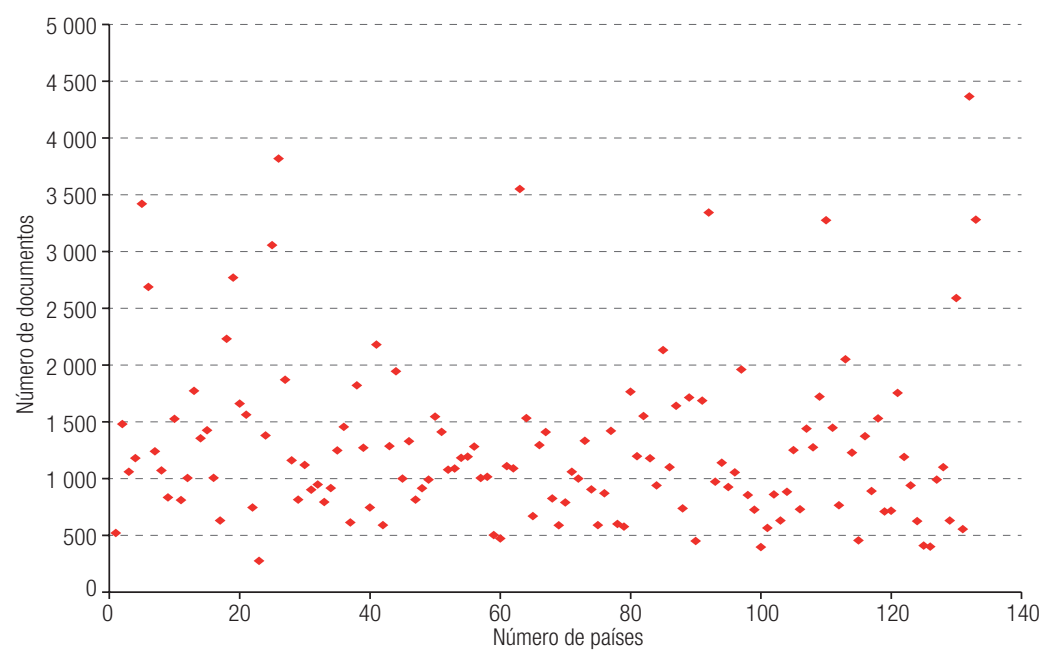

Fuente: Elaboración propia, sobre la base de datos de Banco Mundial, "Datos de libre acceso del Banco Mundial” [en línea] https://datos.bancomundial.org.

a Países cuyas exportaciones e importaciones con Chile se mantuvieron continuas en el período que va de 2006 a 2014. 
El promedio de los costos de exportación de los países del Cono Sur ha aumentado de 966,67 dólares por contenedor, en 2005, a 1.772,9 dólares, en 2014. Se destaca el alza de los costos en la República Bolivariana de Venezuela, el Brasil y Colombia. En 2005, esos países presentaban los menores costos de exportación por contenedor, junto con el Perú, pero en 2007, 2008 y 2009, respectivamente, dichos costos aumentaron de forma drástica. En particular, entre 2012 y 2014 se registra un aumento significativo de los costos de exportación por contenedor en la República Bolivariana de Venezuela (3.490 dólares), el Brasil (2.414 dólares), Colombia (2.355 dólares), el Paraguay (1.850 dólares) y la Argentina (1.650 dólares). En Chile y el Perú se ha logrado mantener los menores costos de exportación por contenedor de América del Sur: en 2014, el costo promedio era de 890 dólares en el Perú y de 980 dólares en Chile.

En los gráficos 3 y 4 se exponen los porcentajes máximos que se aplicaron como arancel en 2009 y 2014 en los países de América del Sur (no se registra información de la República Bolivariana de Venezuela en 2009). En 2014, la República Bolivariana de Venezuela, Colombia y el Ecuador son los países donde se aplicó el mayor arancel, del 160\%, el 98\% y el $86 \%$, respectivamente (OMC/CCI/UNCTAD, 2015). Chile y el Perú son los países en los que se aplicaron los menores aranceles máximos en 2009 y 2014. Chile es el que presenta el indicador más bajo: un 13\% en 2009 y un 6\% en 2014. Los aranceles máximos que se aplicaron en 2014 en los países de América del Sur están por debajo del promedio de los aplicados a nivel mundial (175,3\%), según lo expuesto en el informe Perfiles arancelarios en el mundo (OMC/CCI/UNCTAD, 2015). Los países del mundo donde se aplican los aranceles más altos son Egipto, Fiji, Malasia y Noruega.

Gráfico 3

América del Sur (9 países): impuesto arancelario máximo aplicado, 2009 (En porcentajes)

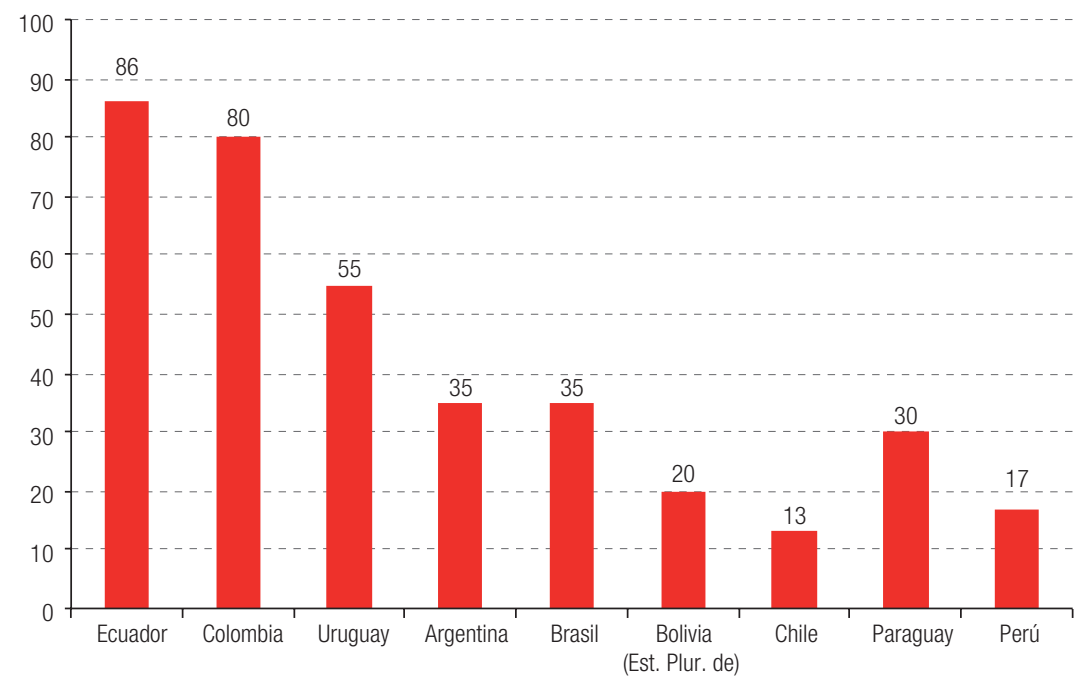

Fuente: Elaboración propia, sobre la base de datos de Organización Mundial del Comercio (OMC)/Centro de Comercio Internacional (CCl)/Conferencia de las Naciones Unidas sobre Comercio y Desarrollo (UNCTAD), Perfiles arancelarios en el mundo, 2010 (UNCTAD/NTO/2010), Ginebra, 2010. 


\section{Gráfico 4}

América del Sur (10 países): impuesto arancelario máximo aplicado, 2014

(En porcentajes y países)

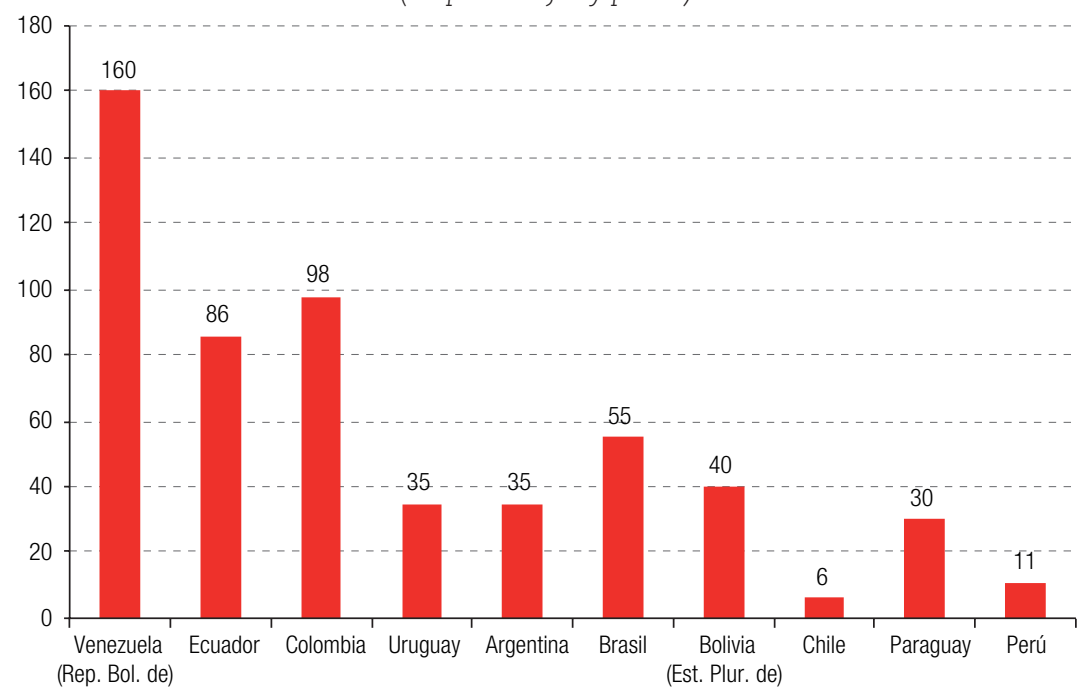

Fuente: Elaboración propia, sobre la base de datos de Organización Mundial del Comercio (OMC)/Centro de Comercio Internacional (CCl)/Conferencia de las Naciones Unidas sobre Comercio y Desarrollo (UNCTAD), Perfiles arancelarios en el mundo, 2015 (UNCTAD/NTO/2015), Ginebra, 2015.

\section{Características del patrón exportador chileno}

En el cuadro 2 se presentan los principales bienes exportables de Chile en 2006 y 2014. Se observa que las exportaciones chilenas en los últimos años siguen concentradas en el cobre y sus derivados, que representan el 57\% del total exportable en 2006 y el 51\% en 2014. Es probable que la disminución de este porcentaje se deba directamente a la baja del precio real del producto, puesto que, en 2006, el precio histórico del cobre refinado real alcanzó 328,2 centavos de dólar por libra y, en 2014, bajó a 299,3 (Sociedad Nacional de Minería)'1.

\section{Cuadro 2}

Chile: principales bienes exportables, 2006 y 2014

(En porcentajes sobre el total)

\begin{tabular}{lrr}
\hline Bienes exportables & 2006 & 2014 \\
\hline Cátodos y secciones de cátodos & 28,84 & 23,02 \\
\hline Minerales de cobre y sus concentrados & 20,45 & 22,2 \\
\hline Cobre para el afino & 4,98 & 3,91 \\
\hline Concentrados & 2,41 & 1,95 \\
\hline Otros & 43,32 & 48,92 \\
\hline
\end{tabular}

Fuente: Elaboración propia, sobre la base de Asociación Latinoamericana de Integración (ALADI), "Estadísticas de comercio exterior", 2016 [en línea] http://consultawebv2.aladi.org/sicoexV2/jsf/comercio_exterior_item_arancelario. seam?cid=10711.

En el cuadro 3 se observan los principales destinos de las exportaciones chilenas, en porcentajes del total, en 2006 y 2014. Se concluye que la diversificación por destino es baja durante el período y que las exportaciones destinadas a China aumentaron un 15,71\% del total exportado en 2006, al 24,55\%, en 2014. Al agrupar por continente los principales destinos de la exportación chilena en 2014, se encuentra que Asia es el principal destino, con un porcentaje levemente inferior al $44,7 \%$ del total exportado.

1 http://www.sonami.cl/index.php?option=com_content\&view=article\&id=224\&ltemid=117. 
Cuadro 3

Chile: principales destinos de las exportaciones, 2006 y 2014

(En porcentajes del total)

\begin{tabular}{lrlr}
\hline País copartícipe & 2006 & País copartícipe & 2014 \\
\hline Estados Unidos & 16,01 & China & 24,55 \\
\hline Japón & 10,81 & Estados Unidos & 11,75 \\
\hline China & 8,84 & Japón & 10,46 \\
\hline Países Bajos & 6,83 & República de Corea & 6,15 \\
\hline República de Corea & 6,09 & Brasil & 5,44 \\
\hline Italia & 5,03 & India & 3,47 \\
\hline Brasil & 4,94 & Países Bajos & 2,90 \\
\hline Otros & 41,45 & Otros & 35,28 \\
\hline
\end{tabular}

Fuente: Elaboración propia, sobre la base de Asociación Latinoamericana de Integración (ALADI), "Estadísticas de comercio exterior", 2016 [en línea] http://consultawebv2.aladi.org/sicoexV2/jsf/comercio_exterior_item_arancelario. seam?cid=10711.

Esta realidad del comercio exterior chileno, que se caracteriza por el extractivismo de recursos naturales y cuyo principal destino exportador es China y otros países asiáticos, se replica en otros países de América Latina. En el estudio de Dingemans y Ross (2012) se evidencia que parte del crecimiento exportador de los países de América Latina ha ocurrido en el margen intensivo y no en el extensivo, por lo que la expansión hacia nuevos productos y socios comerciales ha sido limitada. Meller, Poniachik y Zenteno (2012) indican que el auge de los precios internacionales de los productos básicos en 2011 y 2012 implicó importantes beneficios para los países de América Latina, sin embargo, y en relación con China: "El gran aumento del 'share' [participación] de los commodities [productos básicos] en la canasta exportadora de América Latina, ha hecho resurgir los temores de una especie de regreso de la 'maldición de los recursos naturales'” (Meller, Poniachik y Zenteno, 2012, pág. 2).

Al analizar la realidad de los países de América Latina, en la cual Chile no es una excepción, se observa que el modelo extractivista aplicado con el propósito de insertarse en el comercio mundial ha provocado un desgaste en el crecimiento económico y el desarrollo social.

\section{Especificación del modelo}

En esta investigación se utilizará un modelo gravitacional ampliado, mediante pool y datos de panel estáticos con efectos fijos, aleatorios y dinámicos (Arellano-Bond y Arellano-Blundell), a fin de analizar los efectos de la facilitación del comercio en las exportaciones chilenas durante el período que va de 2006 a 2014. Los primeros autores que utilizaron el modelo gravitacional para analizar el comercio internacional fueron Tinbergen (1962), Pöyhönen (1963) y Linnemann (1966). En los estudios de Anderson (1979), Bergstrand (1985) y Helpman y Krugman (1985), se derivaron ecuaciones gravitacionales de modelos de comercio internacional fundados en la diferenciación del producto y en el rendimiento a escala creciente, y se brindó un sustento teórico riguroso. Mátyás (1997), Cheng y Wall (1999), Bayoumi y Eichengreen (1998), Breuss y Egger (1999), y Egger (2000) proporcionan especificaciones econométricas. En las últimas décadas, el modelo gravitacional es uno de los modelos que más se ha utilizado para analizar los flujos del comercio internacional, la migración o la inversión extranjera, debido a sus propiedades, a su soporte teórico y empírico, y a su flexibilidad y adaptación a diferentes realidades regionales o a la de un país en particular.

Para este estudio, el modelo lineal gravitacional ampliado estará dado por la siguiente ecuación expresada en logaritmos naturales: 


$$
\begin{gathered}
\ln \left(E_{i j t}\right)=\beta_{0}+\mu_{1} \ln y_{i t}+\mu_{2} \ln y_{j t}+\xi_{1} \ln \text { Arance }_{i t}+\xi_{2} \ln \text { Arancel }_{j t} \\
+\alpha_{1} \ln D_{i j}+\phi_{1} \ln \text { Doce }_{i t}+\phi_{2} \ln \text { Doci }_{j t}+\eta_{1} \ln \text { Cose }_{i t}+\eta_{2} \ln \text { Cosi }_{j t} \\
+\nu_{1} \ln T C E_{i t}+\sum_{k} \delta_{h} P_{i j}+u_{i j t}
\end{gathered}
$$

donde?

$i, j$ y $t$ representan el país exportador, el país importador y el año, respectivamente;

$E_{i j}$ representa el flujo de exportación del país $i$ al $j$;

$Y_{i, j}$ es el producto nacional bruto real del país $i$ y $j$, respectivamente;

Arancel $_{i}$, Arancel $_{j}$ representan el promedio ponderado del país $i$ y $j$ correspondiente a la tasa arancelaria aplicada (en porcentajes);

$D_{i j}$ es la distancia geográfica entre las capitales de los países $i$ y $j$ en kilómetros;

Doce es el número de documentos del país exportador que se necesitan para exportar;

Doci es el número de documentos del país importador que se necesitan para importar;

Cose representa el costo de exportación por contenedor, en dólares;

Cosi representa el costo de importación por contenedor, en dólares;

$T C E_{i}$ es el tipo de cambio efectivo del país exportador.

Además, en la ecuación (1) se incluyen variables ficticias indicadas como $\left(\sum_{h} \delta_{h} P_{i j}\right)$, que asumen el valor 1 en caso de presentarse el evento o el valor 0 en caso de que este no se presente. Las variables ficticias son las siguientes:

$F_{i j} \quad$ : frontera común entre el país $i$ y el país $j$;

$I C_{i j} \quad$ : idioma en común entre el país $i$ y el país $j$;

$T L C A N-C L \quad$ : intercambio comercial entre Chile y los países miembros del Tratado de Libre Comercio de América del Norte (TLCAN), es decir, los Estados Unidos, el Canadá y México;

MERCOSUR-CL: intercambio comercial entre Chile y los países miembros del Mercado Común del Sur (MERCOSUR), es decir, la Argentina, el Brasil, el Paraguay, el Uruguay y, desde 2014, la República Bolivariana de Venezuela;

China-Cl

: intercambio comercial entre China y Chile;

$U E-C L$

: intercambio Comercial entre Chile y países miembros de la Unión Europea (UE);

2006-2007

: variable temporal que representa los años 2006 y 2007;

2009

: variable temporal que representa el año 2009, y

2013-2014 : variable temporal que representa los años 2013 y 2014.

Teóricamente, a mayor producto nacional bruto real del país exportador e importador, mayor atracción gravitacional entre ellos, mientras que, a mayor distancia geográfica, menor atracción. Con el número de documentos que se exigen para exportar e importar, el costo de exportación e importación por contenedor, y las tasas arancelarias se procura representar la facilitación comercial y medir sus efectos en el comercio exterior chileno. Los acuerdos comerciales y los tratados de libre

\footnotetext{
2 Las bases de datos que se utilizarán son las siguientes: Asociación Latinoamericana de Integración (ALADI) (www.aladi.org), montos exportados de países de América Latina en millones de dólares FOB y montos importados de países no pertenecientes a Latinoamérica en millones de dólares CIF; www.wcrl.ars.usda.gov/cec/java/lat-long.htm, distancia en kilómetros entre las capitales de los países en estudio; Banco Mundial, datos estadísticos (http://datos.bancomundial.org/).
} 
comercio de Chile con países miembros del TLCAN, el MERCOSUR, la UE y China permitirán medir si dichos acuerdos facilitan el comercio bilateral.

La variable tipo de cambio efectivo del exportador se incluye en la ecuación gravitacional para determinar el impacto que puede tener en el volumen exportado. De acuerdo con Bernat (2015), en la medida en que la producción de un país se caracterice por una baja incidencia de los insumos no transables en su estructura de costos, puede plantearse como hipótesis plausible que la evolución del tipo de cambio real tendrá una influencia escasa en la mayoría de las exportaciones de ese país.

Con la variable ficticia frontera en común se busca diferenciar los efectos fronterizos del comercio con países vecinos del resto del comercio bilateral chileno. Además, con esa variable se medirá el uso mayoritario del transporte terrestre con estos países. Se incluyen asimismo variables ficticias de tiempo que permiten capturar los efectos temporales y las características de esos años, como la crisis de las hipotecas de alto riesgo en 2009 y la baja del precio del cobre en 2013 y 2014.

En este estudio se incluirán 89 países que son socios comerciales, según su pertenencia a una zona geográfica, a saber: 31 países pertenecientes a Europa; 10 a África; 15 a Asia; 7 a Oriente Medio; 2 a América del Norte; 11 a Centroamérica y el Caribe; 11 a América del Sur, y 2 a Oceanía. Dichos países constituían el $91 \%$ de los destinos de exportación de Chile durante el período de estudio. La exclusión de algunos países se debe a la escasez o ausencia de intercambio durante uno o más años consecutivos en dicho período. El total de observaciones por año son 178, lo que representa un total de 1.602 observaciones.

Dado que al aplicar un modelo gravitacional por lo general se usa una muestra grande de países y años, es posible que algunas observaciones de las variables endógenas (como la exportación, la importación o el comercio total) asuman valor 0. A fin de abordar el problema de las observaciones 0 en el comercio, Piani y Kume (2000), Zago de Azevedo (2001) y Wall (2003) las han sustituido por valores pequeños $(0,001)$ en la variable dependiente (monto exportado por los socios comerciales), y Eichengreen e Irwin (1998) y Wall (2003) le han sumado 1 a todas las observaciones. Sin embargo, Santos-Silva y Tenreyro (2006) exponen un problema relacionado con la analogía entre la gravedad newtoniana y el comercio, a saber, que la fuerza gravitacional puede ser muy pequeña, pero nunca es 0 , mientras que el comercio entre varios pares de países puede alcanzar valores 0 . Un problema adicional es el uso de logaritmos en la forma lineal de la ecuación gravitacional. Frente a esto y a los posibles métodos que se pueden emplear para abordar este problema, los autores plantean que sumarle 1 a la variable dependiente o usar un estimador tobit puede dar lugar a estimadores incongruentes.

En este estudio, se siguió una de las recomendaciones que brindan Santos-Silva y Tenreyro (2006) y se optó por dejar una muestra de países cuyo flujo de comercio bilateral con Chile no presentaba observaciones 0 en el período en estudio. Para ello, se revisaron los montos de exportación e importación en detalle y no en miles de dólares, y se excluyeron los países socios comerciales que, en un año o más, presentaban 0 en la variable dependiente. Por consiguiente, la muestra quedó determinada por 89 países que abarcaban el 91\% del monto total exportado por Chile durante el período en estudio. La ecuación (1) se estimará mediante datos de panel estático con efectos aleatorios, donde el intercepto de la regresión será aleatorio $\left(\beta_{0}\right)$ y se considerará como $\beta_{0}=\beta+u_{i}$. Es decir, en vez de considerar a $\beta$ como un intercepto fijo, se proyecta como una variable aleatoria con un valor medio $\beta$ y una desviación aleatoria $u_{i}$ de este valor medio.

La ecuación (1) se adapta para hacer la estimación con datos de panel de efectos fijos y con ello se obtiene la ecuación (2), en la que se excluyen todas las variables fijas que se mantienen a través del período en estudio. Esta estimación permite observar intersecciones de todos los pares de socios comerciales, descartando una constante en común. 


$$
\begin{aligned}
& \ln \left(E_{i j t}\right)=\beta_{i j t}+\mu_{1} \ln y_{i t}+\mu_{2} \ln y_{j t}+\xi_{1} \ln \text { Arancel }_{i t}+\xi_{2} \ln \text { Arancel }_{j t} \\
& +\phi_{1} \ln \text { Doce }_{t}+\phi_{2} \ln \text { Doci }_{t}+\eta_{1} \ln \text { Cose }_{t}+\eta_{2} \ln \text { Cosi }_{t}+\nu_{1} \ln T C E_{i t}+u_{i j t}
\end{aligned}
$$

Donde $\beta_{i j t}$ es un número fijo para cada par de socios comerciales.

Dada la posible endogeneidad del fenómeno en estudio, se recomienda utilizar datos de panel con efectos dinámicos. Arellano y Bover (1990) exponen que uno de los aspectos en que el uso del panel resulta positivo y decisivo respecto a una muestra de corte transversal es la posibilidad de modelizar respuestas dinámicas con microdatos: "Ecuaciones con retardos de variables endógenas y exógenas pueden ser especificadas permitiendo la posibilidad de explicar procesos de ajuste" (Arellano y Bover, 1990, pág. 5).

La ecuación (1) se adapta para hacer la estimación con datos de panel dinámicos (ecuación (3)).

$$
\begin{gathered}
\ln \left(E_{i j t}\right)=\beta_{0}+\beta_{1} \ln \left(E_{i j t-1}\right)+\beta_{1} \ln \left(E_{i j t-2}\right)+\mu_{1} \ln y_{i t}+\mu_{2} \ln y_{j t}+ \\
\xi_{1} \ln \text { Arancel }_{i t}+\xi_{2} \ln \text { Arancel }_{j t}+\alpha_{1} \ln D_{i j t}+\phi_{1} \ln \text { Doce }_{t}+\phi_{2} \ln \text { Doci }_{t} \\
+\eta_{1} \ln _{\text {Cose }}+\eta_{2} \ln \text { Cosi }_{t}+\nu_{1} \ln T C E_{i t}+\sum_{k} \delta_{h} P_{i j}+u_{i j t}
\end{gathered}
$$

Donde $E_{i j t-1}$ es el primer retardo de la variable dependiente y $E_{i j t-2}$ el flujo de exportación del país $i$ al país $j$. Esta ecuación se estimará mediante las técnicas de Arellano-Bond y Arellano-Blundell.

\section{Resultados}

En el cuadro 4 se pueden observar los resultados que se obtuvieron al utilizar datos de panel estáticos con efectos aleatorios y fijos (ecuación (1) y (2), respectivamente), y con efectos dinámicos (ecuación (3), con Arellano-Bond y Arellano-Blundell). Con el propósito de observar las diferencias entre el coeficiente de efectos fijos y el de efectos aleatorios, se aplica la prueba de Hausman y se obtiene Prob> Chi2=0,051. Por consiguiente, se ratifica que el método de efectos aleatorios es más conveniente que el de efectos fijos para explicar el comportamiento chileno en cuanto a la exportación de bienes manufacturados hacia los países en estudio. Sin embargo, el coeficiente de determinación que se alcanza al estimar mediante efectos aleatorios $(0,77)$ es medianamente alto. En la prueba de Durbin, Wu y Hausman se detecta endogeneidad, por lo que se recomienda utilizar el panel dinámico.

En el cuadro 4 se observan los resultados que se obtuvieron al estimar la ecuación (3) mediante datos de panel con efectos dinámicos y dos retardos de la variable dependiente, utilizando las técnicas de Arellano-Bond y de Arellano-Blundell. De acuerdo con los coeficientes estimados mediante datos de panel con efectos dinámicos (ecuación (3), cuadro 4), es posible concluir que la endogeneidad disminuye y concuerda con lo esperado desde el punto de vista teórico. Los resultados indican que el primer retardo de la variable dependiente tiene signo positivo y significativo, mientras que, en el segundo retardo, el coeficiente es negativo y significativo. 
Cuadro 4

Resultados de la estimación de las ecuaciones (1), (2) y (3)

\begin{tabular}{|c|c|c|c|c|}
\hline Variables & $\begin{array}{l}\text { Efectos fijos } \\
\text { (ecuación (2)) }\end{array}$ & $\begin{array}{l}\text { Efectos aleatorios } \\
\text { (ecuación (1)) }\end{array}$ & $\begin{array}{l}\text { Efectos dinámicos } \\
\text { (ecuación (3), } \\
\text { Arellano-Bond) }\end{array}$ & $\begin{array}{c}\text { Efectos } \\
\text { dinámicos } \\
\text { (ecuación (3), } \\
\text { Arellano-Blundell) }\end{array}$ \\
\hline $\operatorname{Ln}(\operatorname{Exp}-1)$ & & & $0,23(0,06)^{\star \star \star}$ & $0,36(0,06)^{\star \star \star}$ \\
\hline $\operatorname{Ln}(\operatorname{Exp}-2)$ & & & $-0,14(0,03)^{\star \star \star}$ & $-0,11(0,03)^{\star \star \star}$ \\
\hline Ln (PNB exportador) & & $1,57(0,08)^{\star \star *}$ & $3,0(0,5)^{\star \star \star}$ & $0,87(0,2)^{\star * \star}$ \\
\hline Ln (PNB importador) & $2,5(0,2)^{\star \star \star}$ & $1,19(0,08)^{\star \star \star}$ & & \\
\hline Ln (distancia geográfica) & & & $-6,5(1,2)^{\star \star \star}$ & \\
\hline \multicolumn{5}{|l|}{$\operatorname{Ln}(D O C E)$} \\
\hline \multicolumn{5}{|l|}{$\operatorname{Ln}(D O C l)$} \\
\hline Ln (costo exportación) & & $-1,2(0,18)^{\star \star \star}$ & $-1,1(0,42)^{\star \star \star}$ & $-1,0(0,4)^{\star \star}$ \\
\hline \multicolumn{5}{|l|}{ Ln (costo de importación) } \\
\hline Ln (arancel exportador) & & $-0,1(0,05)^{\star}$ & & \\
\hline \multicolumn{5}{|l|}{ Ln (arancel importador) } \\
\hline \multicolumn{5}{|l|}{ Ln (tipo de cambio efectivo) } \\
\hline \multicolumn{5}{|l|}{ Frontera en común } \\
\hline Idioma en común & & $2,93(0,32)^{\star \star \star}$ & & \\
\hline TLCAN-CL & & & & $24,6(11,06)^{\star}$ \\
\hline MERCOSUR-CL & & $1,77(0,6)^{\star * *}$ & & $-19,8(7,7)^{\star \star}$ \\
\hline \multicolumn{5}{|l|}{ CHINA-CL } \\
\hline \multicolumn{5}{|l|}{$U E-C L$} \\
\hline Años 2006 y 2007 & & $-0,39(0,07)^{\star \star \star}$ & & \\
\hline Año 2009 & & $-0,22(0,08)^{\star \star \star}$ & & $-0,36(0,06)^{\star \star \star}$ \\
\hline \multicolumn{5}{|l|}{ Años 2013 y 2014} \\
\hline Constante & $-54,4(6,0)^{\star \star \star}$ & $-51,9(3,1)^{\star \star \star}$ & & $-14,4(5,4)^{\star \star \star}$ \\
\hline $\mathrm{R}^{2}$ & 0,18 & 0,77 & & \\
\hline Número de observaciones & 1602 & 1602 & 1602 & 1602 \\
\hline
\end{tabular}

Fuente: Elaboración propia.

Nota 1: Valores con heterocedasticidad corregida.

Nota 2: Nivel de significancia: ${ }^{* \star}=0 \%$ error; ${ }^{* *}=0 \%<\mathrm{P} \leq 2,5 \% ;{ }^{*}=2,5 \%<\mathrm{P} \leq 5 \%$

Nota 3: Los valores entre paréntesis representan el estándar de error.

Se observa que, en las estimaciones mediante efectos aleatorios y efectos dinámicos (Arellano-Bond y Arellano-Blundell), el coeficiente del producto nacional bruto real del exportador es positivo y significativo, y el coeficiente de los costos de exportación es negativo y significativo.

El efecto dinámico mediante la técnica de Arellano-Blundell reduce significativamente la endogeneidad. Se observa que el acuerdo entre el TLCAN y Chile afecta de forma positiva el comercio bilateral chileno, mientras que el acuerdo entre el MERCOSUR y Chile presenta efectos negativos. También se reconoce que la crisis de las hipotecas de alto riesgo tuvo un efecto negativo en el comercio bilateral chileno. No se observa ningún efecto significativo de las variables que representan los documentos que se exigen para exportar e importar, los costos de importación, el arancel del exportador y el importador, el tipo de cambio efectivo, la frontera en común, el idioma en común, el acuerdo entre China y Chile, el acuerdo entre la UE y Chile, y las variables temporales de 2006 y 2007, y 2013 y 2014

\section{Conclusiones y recomendaciones}

Dado que Chile está inserto en la realidad de América Latina, para analizar los efectos de la facilitación comercial en las exportaciones e importaciones chilenas durante el período de 2006 a 2014, en primer lugar se hizo una revisión de casos y evidencias acerca de dicha facilitación en países de América 
Latina, incluido Chile, y se los comparó con países de otros continentes. Además, se analizaron datos estadísticos acerca de la facilitación comercial en Chile y sus principales socios comerciales en cuanto a los documentos requeridos para importar y exportar, los costos de exportación e importación por contenedor, y los impuestos arancelarios máximos aplicados. Luego, se analizó el patrón exportador chileno y sus implicaciones. Finalmente, se especificó un modelo gravitacional y se lo aplicó mediante datos de panel, para analizar los efectos de la facilitación comercial en las exportaciones chilenas.

En los estudios referidos a América Latina se observa una gran preocupación por la excesiva documentación que se exige para exportar e importar, los altos costos que supone exportar un contenedor, la ineficiencia de los servicios logísticos y los procedimientos aduaneros, las escasa infraestructura portuaria y la lenta gestión de la instituciones públicas en general, que afectan el precio del bien exportado y conducen a que el precio pierda competitividad en el país de destino. Las evidencias de los últimos años manifiestan un aumento gradual de los costos de exportación por contenedor en América Latina, principalmente en la República Bolivariana de Venezuela, el Brasil y Colombia. Se reconoce a Chile y el Perú como los únicos países en los que se han mantenido bajos costos de exportación por contenedor que son inferiores al promedio mundial, pero que duplican los que se observan en los países asiáticos.

Esta realidad sugiere la necesidad de llevar a cabo reformas que permitan reducir los procedimientos aduaneros y orientar la inversión pública y privada hacia proyectos de incremento o mejoramiento de la infraestructura portuaria, terrestre y vial que potencie las ventajas comparativas y aumente la competitividad de la región. Esto redundaría en el aumento de las exportaciones de bienes tradicionales y no tradicionales en América del Sur. Sobre la base de las evidencias de Iwanow y Kikpatrick (2007), Jean-François y otros (2007) y Zaki (2015), disminuir la burocracia y los niveles de corrupción percibida, sumado a herramientas tecnológicas, permitiría reducir el tiempo que insume importar y exportar, en particular los días de diligencia en América Latina. Ello bajaría los costos de exportación por contenedor y potenciaría la competitividad de las exportaciones regionales. En las conclusiones de varios estudios, como los de Márquez-Ramos y otros (2007 y 2011), que se centran en el caso de España y América Latina, se señala que reducir los costos de exportación por contenedor y del flete en la cadena de transporte contribuye a aumentar el volumen exportado.

Las exportaciones chilenas siguen estando determinadas por la falta de diversificación, tanto de los bienes exportados como de los países de destino. La alta dependencia del cobre y sus derivados, como bienes exportados principales y predominantes, constituye una vulnerabilidad potencial del comercio y la economía nacional que debe abordarse en el corto plazo. Probablemente, esta dependencia desincentiva la aplicación de medidas de facilitación comercial, lo que afecta la diversificación de los bienes exportables y, por otra parte, condiciona el comportamiento exportador en el año posterior. En varios estudios (Wilson, 2007; Moreira y otros, 2013, entre otros) se indica que, cuanto menores son los costos de exportación, mayor es la producción de bienes manufacturados, y estas evidencias deben incluirse en el debate relativo a las políticas públicas y económicas de Chile cuyo propósito sea fomentar las exportaciones con mayor intensidad tecnológica.

Al estimar el modelo gravitacional de datos de panel estáticos con efectos fijos y aleatorios, los resultados ratifican que es preferible hacer el cálculo empleando datos de panel con efectos aleatorios y dinámicos con dos retardos de la variable dependiente, aunque la estimación mediante datos de panel dinámico (Arellano-Blundell) describe mejor el comportamiento exportador chileno y es coherente con las evidencias y teorías que se han planteado acerca de la facilitación comercial. Estos resultados indican lo siguiente:

- A mayor exportación del año anterior, menor exportación del antepenúltimo año. Esto indica que el comportamiento exportador e importador de Chile es dinámico y fluctuante, con efectos negativos cada dos años, pero con tendencia al alza. También se observa que, cuanto 
mayor sea el producto nacional bruto real del exportador y menor el costo de exportación por contenedor, mayor será el flujo comercial entre Chile y sus socios comerciales. El coeficiente correspondiente al impacto del costo de exportación por contenedor es superior al que se observa en los coeficientes de los retardos y producto nacional bruto real del exportador. Por consiguiente, se considera necesario disminuir el costo de exportación por contenedor para facilitar el comercio chileno. Disminuir este costo implica enfrentar la realidad portuaria chilena e invertir en infraestructura y capacidad portuarias, que son muy limitadas, mejorar los servicios logísticos, concretizar proyectos de infraestructura que optimicen las conexiones terrestres y viales, y reducir los tiempos de espera en puertos y almacenes públicos y privados.

- Las tasas arancelarias promedio del importador y el exportador no afectan el flujo comercial entre Chile y los países en estudio. Una explicación posible es que el arancel ponderado promedio de Chile ha ido disminuyendo y ya era muy bajo durante el período en estudio. Según datos de la Cámara de Comercio de Santiago (CCS, 2016), el arancel efectivo de importación de Chile bajó del 8\% al 2,1\% entre 1999 y 2004, y luego a 1,2\% en 2009, cifra que se ha mantenido hasta 2015.

- $\quad$ El Tratado de Libre Comercio (TLC) firmado entre los países miembros del TLCAN y Chile ha facilitado el intercambio comercial, mientras que el acuerdo de complementación económica con los países miembros del MERCOSUR ha sido negativo para el flujo comercial entre Chile y estos países. Los otros acuerdos, como el celebrado con China y la UE, no presentan significatividad durante el período de estudio. Estos resultados muestran la importancia de los TLC firmados con los miembros del TLCAN, el primero de los cuales fue el del Canadá (1996), luego el de México (1998) y, finalmente, el de los Estados Unidos (2003). En este sentido, la reciente Alianza del Pacífico conformada por Chile, Colombia, México y el Perú podría facilitar el comercio exterior chileno en las próximas décadas. Por otra parte, la integración de Chile con los países miembros del MERCOSUR se vuelve contraproducente para el comercio bilateral chileno. Según García (2013), la Alianza del Pacífico se puede definir como una nueva etapa de integración latinoamericana, en la que se dejan atrás modelos más ideológicos que integracionistas, que se caracterizan por ser cerrados, como es el caso del MERCOSUR. La falta de significatividad del TLC entre Chile y China puede deberse a que es un acuerdo reciente, firmado en octubre de 2006, por lo que su efecto en el comercio bilateral aún no se logra percibir empíricamente. También puede deberse a que el cobre y sus derivados son el principal bien exportado a este país, por lo que no sería el acuerdo el que desvíe o fomente el comercio, sino el producto nacional bruto del exportador, que en este estudio se presenta como un coeficiente positivo y significativo.

- Las variables que representan la distancia geográfica, la frontera en común y el idioma en común no influyen en el proceso exportador e importador de Chile, debido probablemente a que el comercio exportador e importador chileno se concentra en países asiáticos, lo que supone una amplia distancia geográfica y distintos idiomas.

- $\quad$ El tipo de cambio efectivo real del país exportador no presenta significatividad, lo que confirma que las exportaciones chilenas no están sustentadas en la intensidad tecnológica, sino en la extracción de recursos naturales. Este resultado concuerda con lo que plantea Bernat (2015).

- La crisis de las hipotecas de alto riesgo afectó negativamente el comercio bilateral chileno, lo que confirma la vulnerabilidad de las exportaciones chilenas, sobre todo por la dependencia del cobre y sus derivados.

A pesar de que la variable relacionada con el número de documentos exigidos para importar y exportar no resultó ser significativa en el modelo estimado, se sugiere reducir aún más dicho número para aumentar la provisión de infraestructura básica y servicios de infraestructura, como lo sugiere 
Barbero (2010), con el propósito de reducir los costos de exportación por contenedor, incrementar los fondos públicos e incentivar las inversiones privadas que mejoren la calidad de las redes de carreteras internas e internacionales.

Se sugiere aumentar el tamaño de los puertos chilenos para reducir los costos de flete, según lo que plantean Márquez-Ramos y otros (2011) y, conforme a las indicaciones de Salgado y Cea (2012), potenciar los puertos de lquique, San Vicente y Valparaíso, puesto que brindan a los exportadores mayores oportunidades de acceder a cadenas logísticas más adecuadas a sus productos. También se recomienda estudiar la factibilidad de proyectos portuarios nuevos, ya que los puertos del sur poseen mejores condiciones de accesibilidad marítima y podrían atraer más compañías navieras a dicha zona.

Finalmente, se sugiere estructurar una planificación de mediano plazo que garantice la conexión terrestre nacional, sobre todo con líneas férreas, revisar la dotación de personal aduanero, y estudiar programas de capacitación y apoyo orientados al personal público y privado que interviene en el proceso logístico, impulsando políticas de calidad en el desempeño logístico.

\section{Bibliografía}

ALADI (Asociación Latinoamericana de Integración) (2016), "Estadísticas de comercio exterior" [en línea] http://consultawebv2.aladi.org/sicoexV2/jsf/comercio_exterior_item_arancelario.seam?cid=10711.

Álvarez, I., B. Fischer y J. M. Natera (2013), "MERCOSUR: tendencias de internacionalización y capacidades tecnológicas", Revista CEPAL, № 109 (LC/G.2556-P), Santiago, Comisión Económica para América Latina y el Caribe (CEPAL).

Anderson, J. (1979), "A theoretical foundation for the gravity equation", American Economic Review, vol. 69, № 1, Nashville, American Economic Association.

Arellano, M. y O. Bover (1990), "La econometría de datos de panel", Investigaciones Económicas (Segunda Época), vol. 14, № 1, Madrid, Fundación SEPI.

Bacaria-Colom, J., M. Osorio-Caballero y A. Artal-Tur (2013), "Evaluación del Acuerdo de Libre Comercio México-Unión Europea mediante un modelo gravitacional", Economía Mexicana. Nueva Época, № 1 , Ciudad de México, Centro de Investigación y Docencia Económicas (CIDE).

Banco Mundial (2013a), "Publications" [en línea] http://go.worldbank.org/2E22KVHM60. (2013b), "Trade and international integration" [en línea] http://go.worldbank.org/912Y1LS7E0.

__(s/f), "Datos de libre acceso del Banco Mundial" [en línea] https://datos.bancomundial.org/.

Barbero, J. A. (2010), "La logística de cargas en América Latina y el Caribe: una agenda para mejorar su desempeño", Notas Técnicas, № IDB-TN-103, Washington, D.C., Banco Interamericano de Desarrollo (BID).

Batra, G., D. Kaufmann y A. Stone (2003), Investment Climate Around the World: Voices of the Firms from the World Business Environment Survey, Washington, D.C., Banco Mundial.

Bayoumi, T. y B. Eichengreen (1998), "Exchange rate volatility and intervention: implications of the theory of optimum currency areas", Journal of International Economics, vol. 45, № 2, Amsterdam, Elsevier.

Bergstrand, J. (1985), "The gravity equation in international trade: some microeconomic foundations and empirical evidence", The Review of Economics and Statistics, vol. 67, Nㅜ 3, Cambridge, Massachusetts, The MIT Press.

Bernat, G. (2015), "Tipo de cambio real y diversificación productiva en América del Sur", serie Estudios y Perspectivas, № 43 (LC/L.4031), Santiago, Comisión Económica para América Latina y el Caribe (CEPAL).

Breuss, F. y P. Egger (1999), "How reliable are estimations of East-West trade potentials based on crosssection gravity analyses?, Empírica, vol. 26, № 2, Springer.

Cárdenas, M. y C. García (2004), "El modelo gravitacional de comercio y el TLC entre Colombia y Estados Unidos", Documentos de Trabajo, № 27, Bogotá, Fundación para la Educación Superior y el Desarrollo (FEDESARROLLO).

Castro, M. (2010), "Diagnóstico y propuestas para el mejoramiento de los procesos de logística y aduana en la región de Centroamérica y Panamá", Documentos de Proyectos (LC/W.301), Santiago, Comisión Económica para América Latina y el Caribe (CEPAL). 
CCS (Cámara de Comercio de Santiago) (2016), "CCS: arancel efectivo de importación cerró 2015 en 1,2\%", Informe económico, Santiago, 29 de febrero.

Cheng, I. y H. Wall (1999), "Controlling for heterogeneity in gravity models of trade", Working Paper, № 1999010A, St. Louis, Banco de la Reserva Federal de St. Louis.

Cordero, M. (2014), "Logística y facilitación del comercio entre la Comunidad del Caribe y Centroamérica", Documentos de Proyectos, № 612 (LC/W.612), Santiago, Comisión Económica para América Latina y el Caribe (CEPAL).

Cortes, J. M. (2014), “¿Cuál es la razón de los altos costos de una exportación en Colombia?”, Bogotá, Universidad Militar Nueva Granada [en línea] http://repository.unimilitar.edu.co/bitstream/10654/11620/2/ Ensayo\%20final.pdf.

De Souza, M. J. P. y H. L. Burnquist (2011), "Impactos da facilitação de comércio: evidências do modelo gravitacional", Revista de Economia e Sociologia Rural, vol. 49, № 4, Brasilia, Sociedad Brasileña de Economía y Sociología Rural.

Dingemans, A. y C. Ross (2012), "Los acuerdos de libre comercio en América Latina desde 1990: una evaluación de la diversificación de exportaciones", Revista CEPAL, № 108 (LC/G.2549-P), Santiago, Comisión Económica para América Latina y el Caribe (CEPAL).

DjanKov, S., C. Freund y C. Pham (2010), "Trading on time", The Review of Economics and Statistics, vol. 92, $N^{\circ}$ 1, Cambridge, Massachusetts, The MIT Press.

Durso, D. y H. Ochoa (2003), "Áreas monetarias óptimas: ¿está Latinoamérica cerca?" [en línea] http://www. aaep.org.ar/anales/works/works2003/Durso_Ochoa.pdf.

Echeverría, M. (2007), "La facilitación del comercio en las negociaciones comerciales, multilaterales y bilaterales", Documentos de Proyectos, № 166 (LC/W.166), Santiago, Comisión Económica para América Latina y el Caribe (CEPAL).

Egger, P. (2000), "A note on the proper econometric specification of the gravity equation", Economics Letters, vol. 66, No 1, Amsterdam, Elsevier.

Eichengreen, B. y D. Irwin (1998), "The role of history in bilateral trade flows", The Regionalization of the World Economy, J. Frankel (ed.), Chicago, The University of Chicago Press.

Engman, M. (2005), "The economic impact of trade facilitation", OECD Trade Policy Papers, № 21, París, Organización de Cooperación y Desarrollo Económicos (OCDE).

Florensa, L. y otros (2013), "Acuerdos de integración económica en Latinoamérica: efectos sobre los márgenes de comercio", Revista de Economía, vol. 20, № 1 [en línea] http://www.bvrie.gub.uy/local/ File/REVECO/2013/Florensa-Marquez-Ramos-Recalde-Barone.pdf.

Fuentes, N. A. y G. del Castillo (2012), "Reelaboración del modelo multisectorial dinámico para la planeación estratégica de la economía mexicana y simulación del programa de facilitación comercial”, Economía Mexicana. Nueva Época, vol. 21, №1, Ciudad de México, Centro de Investigación y Docencia Económicas.

García, J. (2013), "Alianza del Pacifico: ¿hacia dónde vamos?", Agenda Internacional, vol. 20, №31, Lima, Instituto de Estudios Internacionales (IDEI).

Giacalone, R. (2003), "CAN-MERCOSUR a la sombra del ALCA", Agroalimentaria, vol. 16, № 16, Mérida, Universidad de los Andes.

Helble, M., B. Shepherd y J. Wilson (2007), Transparency and Trade Facilitation in the Asia Pacific: Estimating Gain from Reform, Washington, D.C., Banco Mundial.

Helpman, E. y P. Krugman (1985), Market Structure and Foreign Trade: Increasing Returns, Imperfect Competition, and the International Economy, Cambridge, Massachusetts, The MIT Press.

Hernández, G. (2014), "Una revisión de los efectos del Tratado de Libre Comercio entre Colombia y Estados Unidos", Lecturas de Economía, № 80, Medellín, Universidad de Antioquia.

Hesketh, D. (2010), "Weaknesses in the supply chain: who packed the box", World Customs Journal, vol. 4, № 2 [en línea] http://worldcustomsjournal.org/Archives/Nolume\%204,\%20Number\%202\%20(Sep\%20 2010)/02\%20Hesketh.pdf.

Hummels, D. (2007), "Transportation costs and international trade in the second era of globalization", Journal of Economic Perspectives, vol. 21, №3, Nashville, Asociación Estadounidense de Economía.

Iwanow, T. y C. Kirkpatrick (2009), "Trade facilitation and manufactured exports: is Africa different?", World Development, vol. 37, № 6, Amsterdam, Elsevier.

(2007), "Trade facilitation, regulatory quality and export performance", Journal of International Development, vol. 19, N 6, Wiley.

Izam, M. (2001), "Facilitación del comercio: un concepto urgente para un tema recurrente", serie Comercio Internacional, № 19 (LC/L.1680-P), Santiago, Comisión Económica para América Latina y el Caribe (CEPAL). 
Jaén, A. (2010), "La UNCTAD y la integración regional en África", Nova Africa, № 26, Barcelona.

Jean-François, A. y otros (2007), Connecting to Compete 2007: Trade Logistics in the Global Economy, Washington, D.C., Banco Mundial.

Jordaan, A. (2014), "The impact of trade facilitation factors on South Africa's exports to a selection of African countries", Development Southern Africa, vol. 31, № 4, Taylor \& Francis.

Kamil, H. y A. Ons (2003), "Formación de bloques comerciales regionales y determinantes del comercio bilateral: el caso del MERCOSUR", Documento de Trabajo, № 2/02, Instituto de Economía (IECON) [en línea] http://www.bvrie.gub.uy/local/File/JAE/2003/iees03j3230803.pdf.

Lara, G. e I. Soloaga (2005), "Determinants of migration in México: gravity and spatial econometrics approaches", Documento de Trabajo, Puebla, Universidad de las Américas.

Lewer, J. y M. Sáenz (2004), "Efectos de la liberalización financiera sobre el comercio exterior: modelo gravitacional de Latinoamérica, 1995-99", Estudios Económicos de Desarrollo Internacional, vol. 4, Nㅜ 2.

Linnemann, H. (1966), An Econometric Study of International Trade Flows, Amsterdam, North Holland.

López, L. y F. Fernando (2005), "La eficiencia portuaria colombiana en el contexto latinoamericano y sus efectos en el proceso de negociación con Estados Unidos" [en línea] https://www.researchgate.net/ publication/5006957_La_eficiencia_portuaria_colombiana_en_el_contexto_latinoamericano_y_sus_ efectos_en_el_proceso_de_negociacion_con_Estados_Unidos.

Márquez-Ramos, L. y otros (2011), "'Special issue on Latin American research maritime networks' services structure and maritime trade", Networks and Spatial Economics, vol. 11, № 3, Springer.

(2007), "Determinantes de los costes de transporte marítimos: el caso de las exportaciones españolas", Revista de Economía, № 834.

Martínez-Zarzoso, I. y L. Márquez-Ramos (2008), "The effect of trade facilitation on sectoral trade", The BE Journal of Economic Analysis \& Policy, vol. 8, № 1, De Gruyter.

Martínez-Zarzoso, I. y F. Nowak-Lehmann (2003), "Argumented gravity model: an empirical application to MERCOSUR-European Union trade flows", Journal of Applied Economics, vol. 6, Nㅜㄹ, Buenos Aires, Universidad del CEMA.

Martínez-Zarzoso, I. y C. Suárez-Burguet (2004), "Transport costs and trade: empirical evidence for Latin American imports from the European Union", Research Papers in International Business, Nㅜ 2-04, Londres, Center for International Business Studies.

Mátyás, L. (1997), "Proper econometric specification of the gravity model", The World Economy, vol. 20, $N^{\circ} 3$, Wiley.

Meller, P., D. Poniachik e I. Zenteno (2012), "El impacto de China en América Latina: ¿desindustrialización y no diversificación de exportaciones?" [en línea] http://www.cieplan.cl/media/publicaciones/archivos/317/ Paper_El_Impacto_de_China_en_America_Latina_Desindustrializacion_y_no_Diversificacion_de_ Exportaciones.pdf.

Moïsé, E. y S. Sorescu (2013), "Trade facilitation indicators: the potential impact of trade facilitation on developing countries", OECD Trade Policy Papers, N 144, París, Organización de Cooperación y Desarrollo Económicos (OCDE).

Moreira, M. y otros (2013), Muy lejos para exportar: los costos internos de transporte y las disparidades en las exportaciones regionales en América Latina y el Caribe, Washington, D.C., Banco Interamericano de Desarrollo (BID).

Nicita, A., M. Olarreaga e I. Soloaga (2003), "The region as an export platform to the world? The case of MERCOSUR", Cuadernos de Economía, vol. 40, № 121, Santiago, Pontificia Universidad Católica de Chile.

Nordas, H. K. y otros (2006), "Logistics and time as a trade barrier", OECD Trade Policy Papers, № 35, París, Organización de Cooperación y Desarrollo Económicos (OCDE).

OCDE (Organización de Cooperación y Desarrollo Económicos) (2003), "Trade facilitation reforms in the service of development" (TD/TC/WP(2003)11/FINAL), París.

OMC/CCI/UNCTAD (Organización Mundial del Comercio/Centro de Comercio Internacional/Conferencia de las Naciones Unidas sobre Comercio y Desarrollo) (2015), Perfiles arancelarios en el mundo, 2015 (UNCTAD/NTO/2010), Ginebra. (2010), Perfiles arancelarios en el mundo, 2010 (UNCTAD/WTO/2010), Ginebra.

Piani, G. y H. Kume (2000), "Fluxos bilaterais de comércio e blocos regionais: uma aplicação do modelo gravitacional", Texto para Discussão, № 749, Río de Janeiro, Instituto de Investigación Económica Aplicada (IPEA).

Portugal-Pérez, A. y J. S. Wilson (2012), "Export performance and trade facilitation reform: hard and soft infrastructure", World Development, vol. 40, № 7, Amsterdam, Elsevier. 
Pöyhönen, P. (1963), "A tentative model for the volume of trade between countries", Weltwirtschaftliches Archiv, $N^{\circ}$ 90, Springer.

Ramos Martínez, A. y otros (2012), "Marcos teóricos para el análisis de las zonas de procesamiento de exportaciones en América Latina y el Caribe", Nota Técnica, N TN-432, Washington, D.C., Banco Interamericano de Desarrollo (BID).

Sá Porto, P. y C. Azzoni (2007), "How international integration affects the exports of Brazilian states", Revista de Economía Mundial, vol. 17, Huelva, Sociedad de Economía Mundial.

Salgado, O. y P. Cea (2012), "Análisis de la conectividad externa de los puertos de Chile como un factor de competitividad", Ingeniare. Revista Chilena de Ingeniería, vol. 20, № 1, Arica, Universidad de Tarapacá.

Sandberg, H., J. Seale y T. Taylor (2006), "History, regionalism and CARICOM trade: a gravity model analysis", Journal of Development Studies, vol. 42, № 5, Taylor \& Francis.

Sant'Anna, V. P. y M. J. P. de Souza (2014), "Uma análise do impacto da facilitação de comércio sobre as exportações brasileiras", Anais do XLI Encontro Nacional de Economia, № 123, Asociación Nacional de Centros de Posgrado en Economía (ANPEC).

Santos-Silva, J. M. C. y S. Tenreyro (2006), "The log of gravity", Review of Economics and Statistics, vol. 88, $N^{\circ} 4$, Cambridge, Massachusetts, The MIT Press.

Serrano, R. y V. Pinilla (2008), "Comercio agrario latinoamericano, 1963-2000: aplicación de la ecuación gravitacional para flujos desagregados de comercio", Documento de Trabajo, № 412, Fundación de las Cajas de Ahorro (FUNCAS).

Servín, M. (2008), "Facilitando el comercio entre el Paraguay y la Unión Europea” [en línea] https://eulacfoundation. org/es/system/files/FACILITANDO\%20EL\%20COMERCIO\%20ENTRE\%2OPARAGUAY\%20Y\%20LA\%20 UE.pdf.

Shepherd, B. (2016), "Did APEC's trade facilitation action plans deliver the goods?", Journal of Asian Economics, vol. 43, Amsterdam, Elsevier.

Shepherd, B. y J. S. Wilson (2006), "Road infrastructure in Europe and Central Asia: does network quality affect trade?", Policy Research Working Paper, № 4104, Washington, D.C., Banco Mundial.

Stark, C. (2011), "Agenda estratégica público-privada de cooperación regional para la facilitación del comercio y la inserción internacional: El Salvador y Guatemala", Documentos de Proyectos (LC/W.374), Santiago, Comisión Económica para América Latina y el Caribe (CEPAL).

Tinbergen, J. (1962), Shaping the World Economy: Suggestions for an International Economic Policy, Nueva York, Twentieth Century Fund.

Ueki, Y. (2015), "Trade cost and exportation: a comparison between enterprises in Southeast Asia and Latin America", Journal of Business Research, vol. 68, № 4, Amsterdam, Elsevier.

Ueki, Y., M. Tsuji y R. Cárcamo (2005), "Tecnología de la información y las comunicaciones (TIC) para el fomento de las pymes exportadoras en América Latina y Asia oriental", Documentos de Proyectos (LC/W.33), Santiago, Comisión Económica para América Latina y el Caribe (CEPAL).

Valenzuela-Klagges, B. (2011), "Comercio y calidad institucional en el crecimiento económico del cono sur americano: cuestiones y desafíos", Revista Pilquen-Sección Ciencias Sociales, № 14, Viedma, Universidad Nacional del Comahue.

Valenzuela-Klagges, B. y L. E. Espinoza-Brito (2015), "Regionalismo latinoamericano y comercio bilateral", Revista Pilquen-Sección Ciencias Sociales, vol. 18, № 2, Viedma, Universidad Nacional del Comahue.

Vallejo, H. y C. Aguilar (2004), "Integración regional y atracción de inversión extranjera directa: el caso de América Latina", Revista Desarrollo y Sociedad, Bogotá, Universidad de los Andes.

Wall, H. (2003), "TLCAN and the geography of North American trade", Review, St. Louis, Banco de la Reserva Federal de St. Louis.

Wilson, N. (2007), "Examining the trade effect of certain customs and administrative procedures", OECD Trade Policy Papers, № 42, París, Organización de Cooperación y Desarrollo Económicos (OCDE).

Wilson, J. S., C. Man y T. Otsuki (2003), "Trade facilitation and economic development: a new approach to quantifying the impact”, The World Bank Economic Review, vol. 17, №3, Oxford University Press.

Zago de Azevedo, A. (2001), "What Has Been the Real Effect of MERCOSUR on Trade? A Gravity Model Approach", tesis [en línea] http://www8.ufrgs.br/ppge/pcientifica/2002_01.pdf.

Zaki, C. (2015), "How does trade facilitation affect international trade?", European Journal of Development Research, vol. 27, № 1.

Zamora, A. y V. Sierens (2014), "Competitividad de los servicios logísticos del comercio exterior en la Cuenca del Pacífico", PORTES, Revista Mexicana de Estudios sobre la Cuenca del Pacífico, vol. 8, № 15, Colima, Universidad de Colima. 